\title{
Differential expression profiles of long noncoding RNA and mRNA in colorectal cancer tissues from patients with lung metastasis
}

\author{
RUO SHU ${ }^{1,2^{*}}, Y_{U} \mathrm{XU}^{1,3^{*}}$, YAN TIAN $^{2,3}$, YUJIAN ZENG $^{1,3}$, LIANG SUN $^{2,3}$, \\ FANGYOU GONG ${ }^{2,3}$, YI LEI ${ }^{2,3}, \mathrm{KUNHUA} \mathrm{WANG}^{1-3}$ and HUAYOU LUO ${ }^{1-3}$ \\ ${ }^{1}$ Department of Gastrointestinal and Hernia Surgery, The First Affiliated Hospital of Kunming Medical University; \\ ${ }^{2}$ Kunming Engineering Technology Center of Digestive Disease; ${ }^{3}$ Yunnan Institute of Digestive Disease, \\ The First Affiliated Hospital of Kunming Medical University, Kunming, Yunnan 650032, P.R. China
}

Received January 19, 2017; Accepted November 21, 2017

DOI: $10.3892 / \mathrm{mmr} .2018 .8576$

\begin{abstract}
Lungs are the most common extra-abdominal site of metastasis of colorectal cancer (CRC), in which long noncoding RNA (IncRNA) may serve a role. In the present study, a high-throughput microarray assay was performed to detect lncRNA expression and identify novel targets for further study of lung metastasis in CRC. In the CRC tissues from patients with lung metastasis, 7,632 lncRNA (3,574 upregulated and 4,058 downregulated) and 6,185 mRNA (3,394 upregulated and 2,791 downregulated) were detected to be differentially expressed with a fold change $\geq 2$ and $\mathrm{P}<0.05$ compared with the CRC tissues without metastasis. A total of six differentially regulated lncRNA were confirmed by reverse transcription-quantitative polymerase chain reaction in 20 pairs of CRC samples. Furthermore, gene ontology and pathway analysis were conducted to predict the possible roles of the identified mRNA. The upregulated mRNA were associated with cell division (biological processes), protein kinase B binding (molecular functions) and cellular components. The downregulated mRNA were associated with cell adhesion, platelet-derived growth factor binding and membrane components. Pathway analysis determined that the upregulated mRNA were associated with the Wnt signaling pathway in the CRC tissues from patients with
\end{abstract}

Correspondence to: Dr Huayou Luo, Department of Gastrointestinal and Hernia Surgery, The First Affiliated Hospital of Kunming Medical University, 295 Xichang Road, Kunming, Yunnan 650032, P.R. China

E-mail: 1677546296@qq.com

Dr Kunhua Wang, Yunnan Institute of Digestive Disease, The First Affiliated Hospital of Kunming Medical University, 295 Xichang Road, Kunming, Yunnan 650032, P.R. China

E-mail: kunhuawang1@163.com

${ }^{*}$ Contributed equally

Key words: colorectal cancer, lung metastasis, long noncoding RNA, biological process, molecular function, cellular component lung metastasis, while the downregulated mRNA were associated with the phosphoinositide 3-kinase/Akt signaling pathway. The results of the present study suggested that differentially expressed lncRNA may be associated with lung metastasis and may provide insights into the biology and prevention of lung metastasis.

\section{Introduction}

Colorectal cancer (CRC) is one of the most common malignant tumors and the third leading cause of cancer-associated mortality worldwide (1). Currently, resection and chemotherapy are commonly used to improve the outcomes in patients with $\mathrm{CRC}$; however, metastasis is an obstacle to successful treatment of CRC (2). Among patients with CRC, 50\% eventually develop distant metastases, resulting in poor outcomes even when primary tumors are resectable (3). Distant metastasis is the predominant cause of death in patients with CRC (4). Lungs are the most common extra-abdominal site of metastasis (5). Diagnosis of lung metastasis in patients with CRC remains dependent on radiology and biopsy (6,7). Radiology-guided biopsy has demonstrated limited sensitivity (8). Therefore, investigation of novel biomarkers and molecular mechanisms underlying metastatic progression in CRC is necessary for improving the survival of patients with $\mathrm{CRC}$, and may be beneficial for earlier diagnosis and treatment of patients with lung metastasis.

Only $\sim 2 \%$ of human genome sequences encode proteins, while the remainder is divided into two groups according the length of the sequence: Short noncoding RNA (<200 nucleotides); and long noncoding RNA (lncRNA; $>200$ nucleotides) $(9,10)$. IncRNA regulate gene expression on transcriptional or post-transcriptional levels $(11,12)$. Currently, an increasing number of studies have demonstrated that lncRNA serve important roles in cancer progression and metastasis as well as cellular processes, including cell proliferation and apoptosis (13-15). Furthermore, it has been reported that many lncRNA serve roles in the regulation of CRC (16). However, biological and pathological functions of the majority of IncRNA in patients with CRC and lung metastasis remain to be elucidated.

Therefore, in the present study, differentially expressed lncRNA and mRNA in tissues from patients with CRC and 
with or without lung metastasis were investigated in order to identify novel diagnostic and prognostic markers for patients with CRC and lung metastasis.

\section{Materials and methods}

Ethical approval. All samples were collected from patients in the Department of Gastrointestinal Surgery, The First Affiliated Hospital of Kunming Medical University (Kunming, China) between January 2013 and December 2015. The project was reviewed and approved by the Ethics Committee of The First Affiliated Hospital of Kunming Medical University. All patients included in the present study provided written informed consent prior to the surgery.

Samples. A total of 6 primary CRC samples were obtained from 3 male patients with $\mathrm{CRC}$ and lung metastasis $(\mathrm{CRC}+\mathrm{m})($ age, $66.33 \pm 7.37$ years) and 3 male patients with CRC without metastasis (CRC - m) (age, 64.67 \pm 11.93 years). All patients were histologically confirmed to have colorectal cancer and did not receive any other forms of therapy at the time of enrollment. At the time of surgery, all tissue samples were immediately frozen in liquid nitrogen and stored at $-80^{\circ} \mathrm{C}$ for further use.

RNA extraction and quality control. Total RNA was extracted from each sample using a homogenizer and TRIzol regent (Thermo Fisher Scientific, Inc., Waltham, MA, USA), according to the manufacturer's protocol. Subsequently, the quantity and quality of purified RNA were assessed by NanoDrop ND-1000 (Thermo Fisher Scientific, Inc.). The integrity of RNA was assessed by electrophoresis on a denaturing $1.5 \%$ agarose gel and staining with ethidium bromide for $30 \mathrm{~min}$ at $60^{\circ} \mathrm{C}$.

Microarray analysis. Sample labeling and human lncRNA array hybridization (Human LncRNA Expression Microarray v4.0, Arraystar, Inc., Rockville, MD, USA) were performed according to the manufacturer's protocol. Total RNA were digested with RNase R (Epicentre; Illumina, Inc., San Diego, CA, USA) to remove linear RNA and enrich circular (circ)RNA. The enriched circRNA were amplified and transcribed into fluorescent lncRNA using random primers (Arraystar Super RNA Labeling kit; Arraystar, Inc.). Labeled IncRNA were purified by RNeasy Mini Kit (Qiagen, Inc., Valencia, CA, USA). The concentration and specific activity of the labeled lncRNA (pmol Cy3/ $\mu \mathrm{g}$ lncRNA) were measured by NanoDrop ND-1000. A total of $1 \mu \mathrm{g}$ of each labeled lncRNA was fragmented by adding $5 \mu \mathrm{l} 10 \mathrm{X}$ blocking agent and $1 \mu \mathrm{l}$ 25X fragmentation buffer (Arraystar Super RNA Labeling kit; Arraystar, Inc.) and the mixture was heated at $60^{\circ} \mathrm{C}$ for 30 min. Finally, $25 \mu 12 \mathrm{X}$ hybridization buffer (GE Healthcare, Chicago, IL, USA) was added to dilute the labeled lncRNA. A total of $50 \mu \mathrm{l}$ hybridization solution was applied onto a gasket slide and assembled to the lncRNA expression microarray slide. The slides were incubated for $17 \mathrm{~h}$ at $65^{\circ} \mathrm{C}$ in an Agilent Hybridization Oven (Agilent Technologies, Inc., Santa Clara, CA, USA). The hybridized arrays were washed using Agilent wash buffer 1 and Agilent wash buffer 2 (Agilent Technologies, Inc., Santa Clara, CA, USA), fixed and scanned using the Agilent Scanner G2505C (Agilent Technologies, Inc.).
Data analysis. Scanned images were imported to the Feature Extraction software (version 11.0.1.1) for raw data extraction (Agilent Technologies, Inc.). When comparing two groups of profile differences (such as metastasis vs. non-metastasis), the fold change (i.e., the ratio of group averages) between the groups of each IncRNA was computed. A t-test was used to determine the differences between two groups. The lncRNA demonstrating fold changes $\geq 2$ and $\mathrm{P}<0.05$ compared with the control were selected as differentially expressed.

Reverse transcription-quantitative polymerase chain reaction (RT-qPCR) validation. A total of 20 pairs of primary CRC samples obtained from patients with $\mathrm{CRC}+\mathrm{m}$ and patients with CRC - $\mathrm{m}$ were used for validation of the results. Following extraction of total RNA, the RNA were reverse-transcribed to cDNA using 5x HiScript ${ }^{\circledR}$ II qRT SuperMix II (R223-01, Vazyme, Piscataway, NJ, USA) according to the manufacturer's protocol. Subsequently, qPCR was performed in $10 \mu \mathrm{l}$ reactions, including $0.5 \mu \mathrm{l}$ PCR forward primer $(10 \mu \mathrm{M}), 0.5 \mu \mathrm{l}$ PCR reverse primer $(10 \mu \mathrm{M}), 2 \mu \mathrm{l}$ cDNA, $5 \mu \mathrm{l} 2 \mathrm{x}$ QuantiFast $^{\circledR}$ SYBR $^{\circledR}$ Green PCR Master Mix (204054, Qiagen, Inc.) and $2 \mu \mathrm{l}$ double distilled water. The following thermocycling conditions were used for qPCR: Initial denaturation at $95^{\circ} \mathrm{C}$ for $10 \mathrm{~min}$; followed by final extension of 40 cycles of $95^{\circ} \mathrm{C}$ for $10 \mathrm{sec}$ and $60^{\circ} \mathrm{C}$ for $60 \mathrm{sec} . \beta$-actin served as a reference gene. Relative expression levels of each lncRNA was calculated using the $2^{-\Delta \Delta \mathrm{Cq}}$ method (17). The statistical significance of the difference was estimated by a t-test using SPSS v20.0 (IBM Corp., Armonk, NY, USA); $\mathrm{P}<0.05$ was considered to indicate a statistically significant difference. The primers used in the present study were as follows: HOXA distal transcript antisense RNA (HOTTIP) forward (F), 5'-CCTAAAGCC ACGCTTCTTTG-3' and reverse (R), 5'-TGCAGGCTGGAG ATCCTACT-3'; RP11-79H23.3 F, 5'-GCAAGGAGAGTAATG CTGGA-3' and R, 5'-CAATGAGGATGAGAAGAGGTC-3'; urothelial cancer associated 1 (UCA1) F, 5'-GTCAACGGA TTTGGTCTGTATT-3' and R, 5'-AGTCTTCTGGGTGGC AGTGAT-3'; metastasis asisassociated lung adenocarcinoma transcript1 (MALAT1) F, 5'-GGTAACGATGGTGTCGAG GTC-3' and R, 5'-CCAGCATTACAGTTCTTGAACATG-3'; LOC100507661 F, 5-CTCGGATCCTACCATCATGGCTC ACTGCAACCTC-3' and R, 5'-CCCTCTAGCGCCGCT TTT TATGCATCA AAA ATAAAGGTG-3'; maternally expressed 3 (MEG3) F, 5'-CTGCCCATCTACACCTCA CG-3' and R, 5'-CTCTCCGCCGTCTGCGCTAGGGGCT-3'; and $\beta$-actin F, 5'-AGCACAGAGCCTCGCCTTTG-3' and R, 5'-CTTCTGACCCATGCCCACCA-3'.

Gene ontology (GO) analysis. GO (geneontology.org) was used for functional analysis in order to categorize genes and gene products into the following categories: i) Implicated in biological processes (BP); ii) demonstrating a molecular function (MF); and iii) associated with cellular components (CC). GO was used to analyze biological functions of associated lncRNA and gene targets. The GO term enrichment technique used the $\mathrm{P}<0.05$ threshold to assign differentially expressed lncRNA to target genes.

Kyoto Encyclopedia of Genes and Genomes (KEGG) pathway analysis. Pathway analysis is a functional analysis 
of genes that allows for construction of network datasets (http://www.genome.jp/kegg/pathway.html). KEGG analysis allowed for determination of biological pathways enriched with differentially expressed genes and lncRNA. P $<0.05$ was considered to indicate a statistically significant difference in gene expression.

\section{Results}

Differentially expressed IncRNA and $m R N A$ in tissues of patients with $C R C+m$. A genome-wide analysis was performed to profile differences in IncRNA and mRNA expression between CRC tissues from patients with CRC $+m$ and CRC - $m$ (Figs. 1 and 2). A total of 7,632 lncRNA and 6,185 mRNA were identified to be differentially expressed with a fold change $\geq 2$ and $\mathrm{P}<0.05$, including 3,574 upregulated (Fig. 1A) and 4,058 downregulated (Fig. 1B) lncRNA. A total of 3,394 and 2,791 mRNA were upregulated (Fig. 2A) and downregulated (Fig. 2B), respectively, in the $\mathrm{CRC}+\mathrm{m}$ group compared with the CRC - $\mathrm{m}$ group.

Validation of deregulated lncRNA. A total of six lncRNA were selected for RT-qPCR verification of microarray results in 20 pairs of CRC samples. The RT-qPCR assay demonstrated that the transcription of IncRNA HOTTIP, RP11-79H23.3, UCA1, MALAT1 and LOC100507661 was significantly upregulated, while the transcription of MEG3 was significantly downregulated in the $\mathrm{CRC}+\mathrm{m}$ group, compared with in the CRC - $\mathrm{m}$ group (Fig. 3A). These results were consistent with the microarray assay (Fig. 3B).

Analysis of upregulated mRNA implicated in BPs. Based on the number of upregulated mRNA implicated in BPs, the 10 most enriched BPs were classified (Fig. 4A). The classification included 2,243, 2,042, 1,963, 1,804, 1,762, 1,736, 1,057, 957, 949 and 900 mRNA involved in single-organism processes, single-organism cellular processes, metabolic processes, organic substance metabolic processes, primary metabolic processes, cellular metabolic processes, single-organism metabolic processes, cellular component organization or biogenesis, organization of cellular components and developmental processes, respectively. A total of 10 most represented BPs were selected based on the enrichment score, including mitotic cell cycle, cell cycle processes, cell cycle, mitotic cell cycle processes, nuclear division, organelle fission, mitotic nuclear division, cell division, organelle organization and DNA metabolic processes (Fig. 4B). The following 10 most represented BPs were also selected based on fold enrichment: Negative regulation of hematopoietic progenitor cell differentiation, chromatin remodeling at centromeres, DNA strand elongation involved in DNA replication, telomere maintenance via semi-conservative replication, centromere protein A (CENP-A) containing nucleosome assembly, CENP-A containing chromatin organization, nucleotide-excision repair, DNA gap filling, DNA strand elongation, telomere maintenance via recombination and pro-B cell differentiation (Fig. 4C).

Analysis of downregulated mRNA implicated in BPs. Based on the number of downregulated mRNA associated with
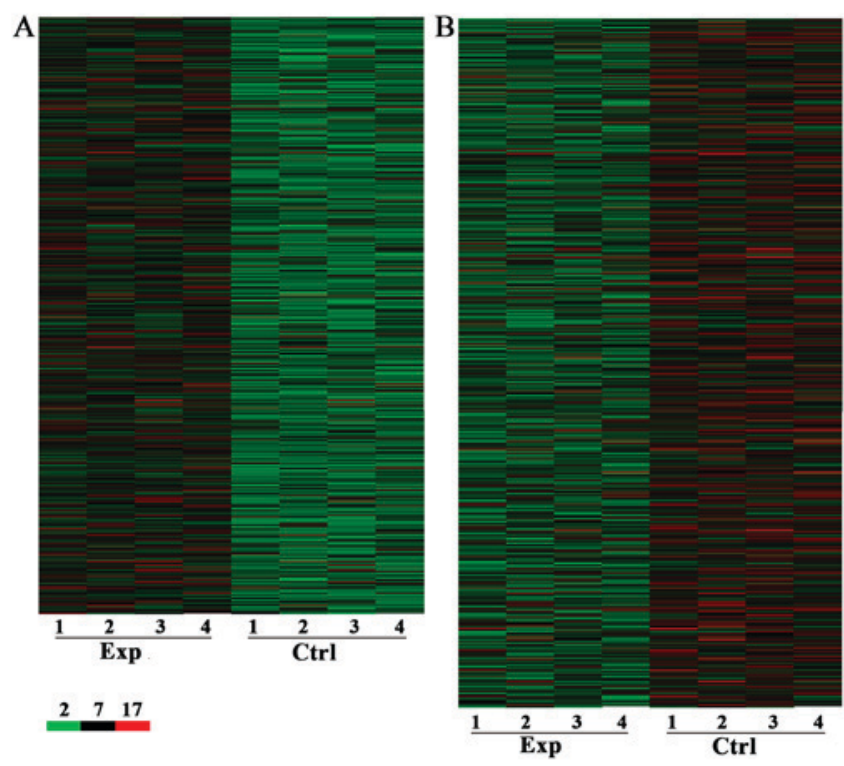

Figure 1. Differentially expressed lncRNA between patients with colorectal cancer with (Exp) or without (Ctrl) lung metastasis. (A) Heat map of upregulated lncRNA. (B) Heat map of downregulated lncRNA. Each column represents one sample. lncRNA, long noncoding RNA; Exp, experimental group; Ctrl, control.
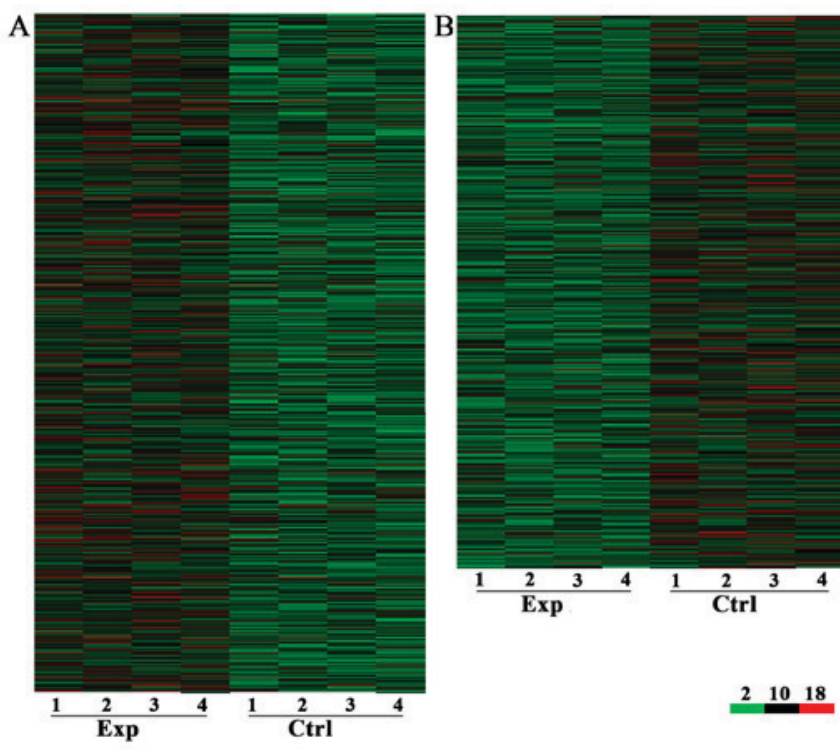

Figure 2. Differentially expressed mRNA between patients with colorectal cancer with (Exp) or without (Ctrl) lung metastasis. (A) Heat map of upregulated mRNA. (B) Heap map of downregulated mRNA. Each column represents one sample. Exp, experimental group; Ctrl, control.

BPs, the 10 most enriched BPs were classified in the present study (Fig. 5A). The BPs included 1,813, 1,636, 1,480, 1,398, $1,326,1,167,1,053,1,033,939$ and 905 mRNA involved in single-organism processes, single-organism cellular processes, biological regulations, regulation of biological processes, regulation of cellular processes, response to stimuli, multicellular organism-associated processes, single-multicellular organism-associated processes, cellular response to stimuli and cell communication, respectively. 


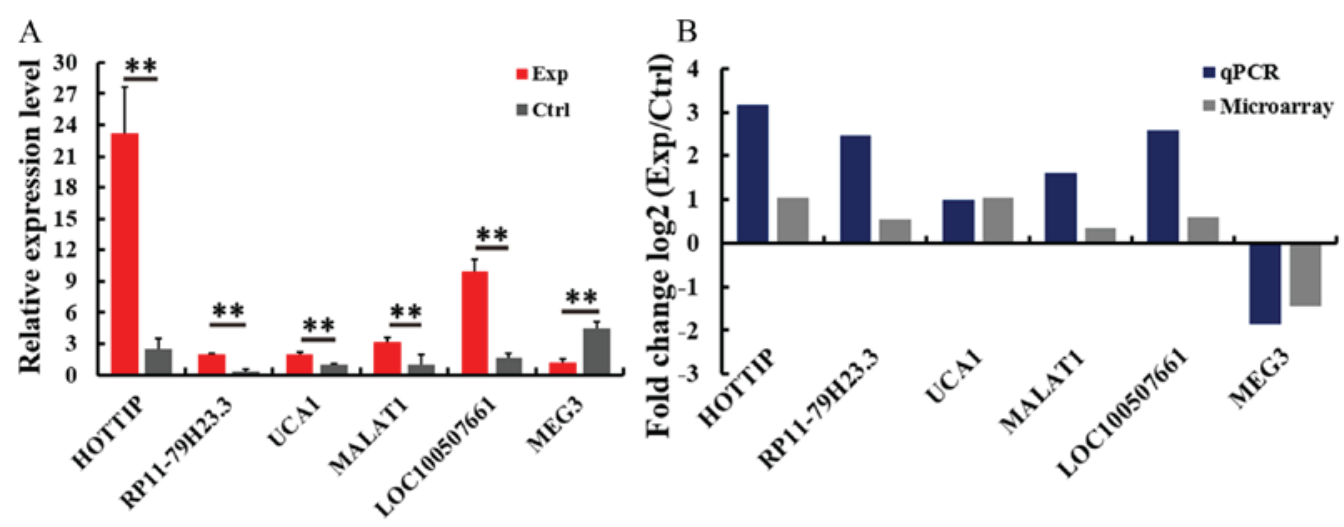

Figure 3. Validation of the microarray assay of differentially expressed lncRNA using RT-qPCR. (A) Comparative expression of lncRNA between the Exp (CRC with lung metastasis) and the $\mathrm{Ctrl}$ (CRC without lung metastasis) group. The data are presented as the mean + standard deviation. ${ }^{* * *} \mathrm{P}<0.05$ as indicated. (B) Comparison of the results obtained by microarray with the results obtained by RT-qPCR. RT-qPCR, reverse transcription-quantitative polymerase chain reaction; CRC, colorectal cancer; lncRNA, long noncoding RNA; Exp, experimental; Ctrl, control; HOTTIP, HOXA distal transcript antisense RNA; UCA1, urothelial cancer associated 1; MALAT1, metastasis associated lung adenocarcinoma transcript 1; MEG3, maternally expressed 3.

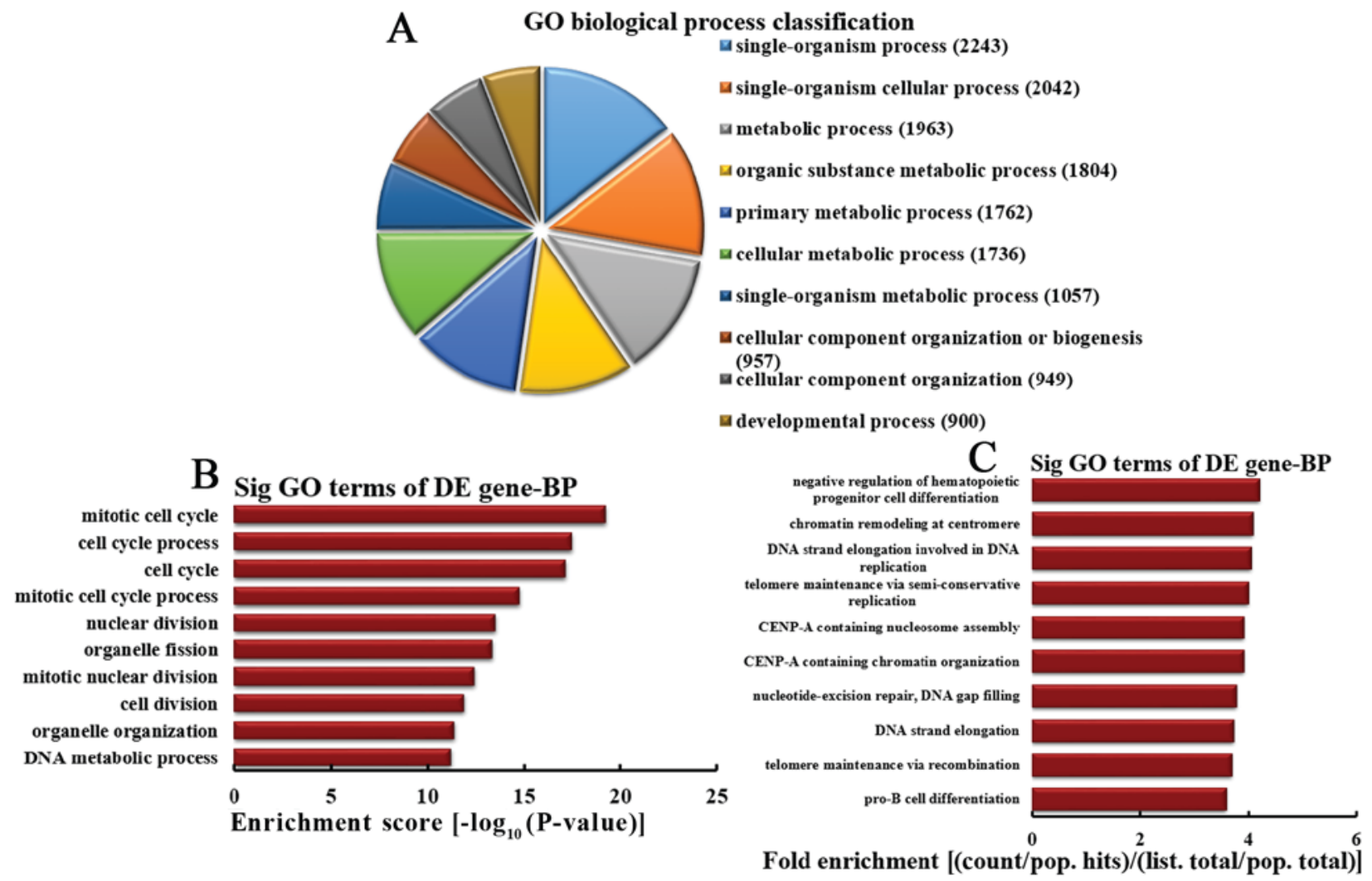

Figure 4. Analysis of upregulated mRNA involved in BPs. (A) BP classification based on the number of DE mRNA. (B) The most represented BPs based on the enrichment score. (C) The most represented BPs according to the fold enrichment. BP, biological process; CENP-A, centromere protein A; GO, gene ontology; DE, differentially expressed; Sig, significant.

Based on the enrichment score, the 10 most enriched BPs were selected, as follows: System development, biological adhesion, cell adhesion, multicellular organismal development, single-organism developmental process, developmental process, anatomical structure development, extracellular matrix organization, extracellular structure organization, and cardiovascular and circulatory system development (Fig. 5B). Based on fold enrichment analysis, the 10 most represented BPs were selected, including dermatan sulfate proteoglycan biosynthetic processes, dermatan sulfate biosynthetic processes, dermatan sulfate proteoglycan metabolic processes, dermatan sulfate metabolic processes, facial nerve morphogenesis, bone trabecula formation, negative regulation of monooxygenase activity, collagen fibril organization, facial nerve development and protein heterotrimerization (Fig. 5C).

Analysis of upregulated mRNA implicated in MFs. Based on the number of upregulated mRNA serving MFs, the 10 most enriched MFs were selected (Fig. 6A). The categories 

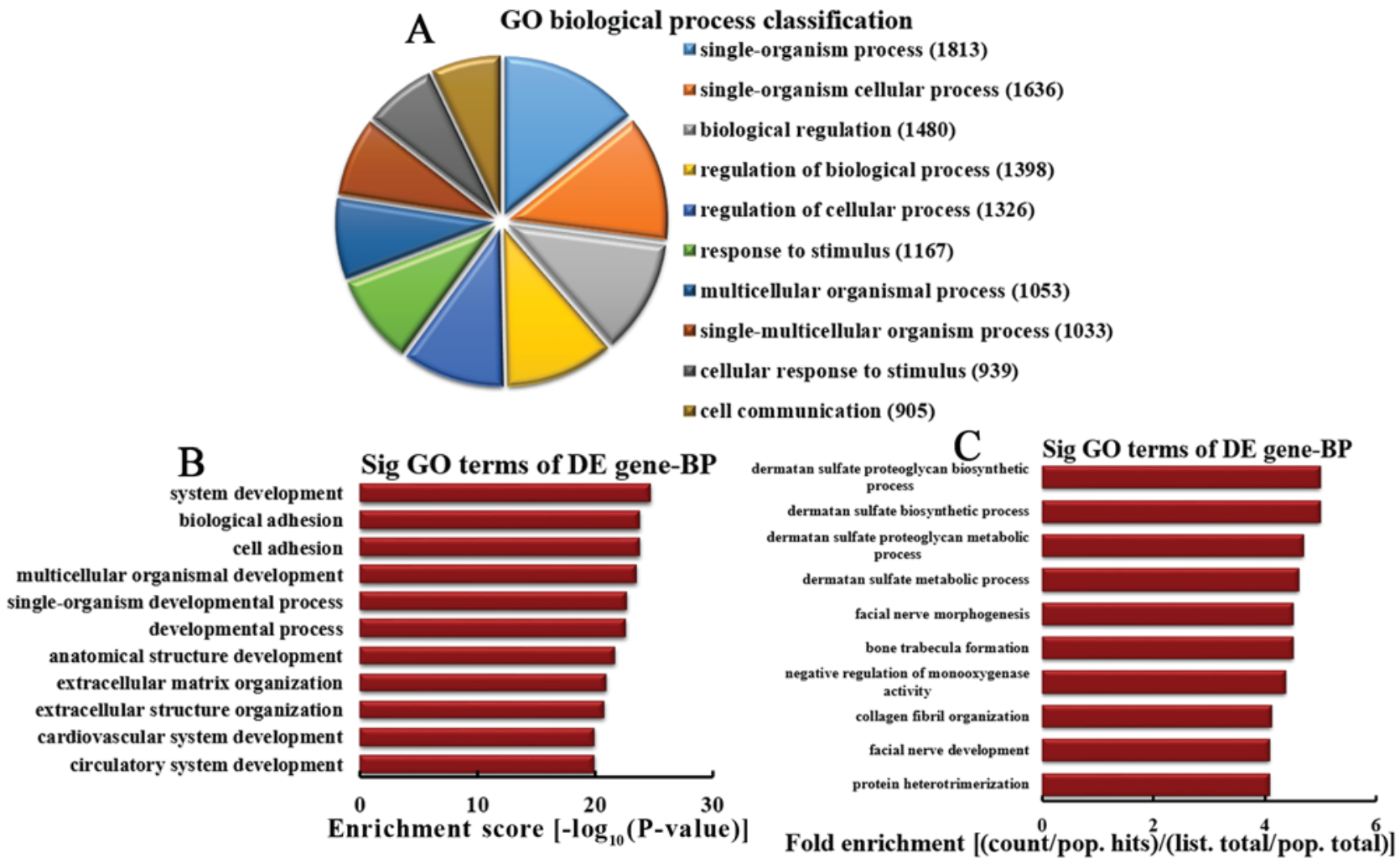

Figure 5. Analysis of downregulated mRNA involved in BPs. (A) BP classification based on the number of DE mRNA. (B) The most represented BPs based on the enrichment score. (C) The most represented BPs according to the fold enrichment. BP, biological process; GO, gene ontology; DE, differentially expressed; Sig, significant.

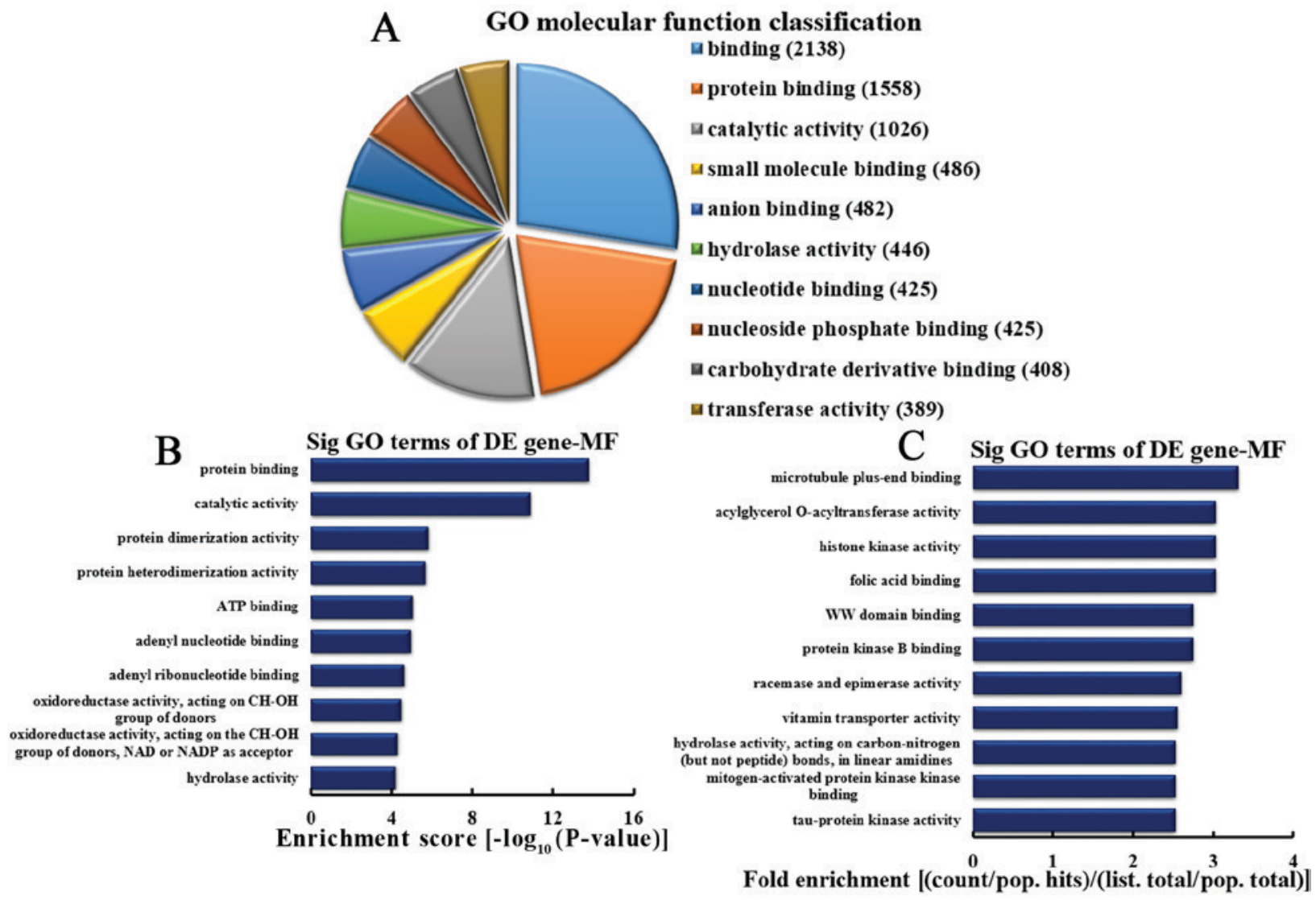

Figure 6. Analysis of upregulated mRNA involved in MFs. (A) MF classification based on the number of DE mRNA. (B) The most represented MFs based on the enrichment score. (C) The most represented MFs according to the fold enrichment. MF, molecular function; GO, gene ontology; DE, differentially expressed; Sig, significant. 


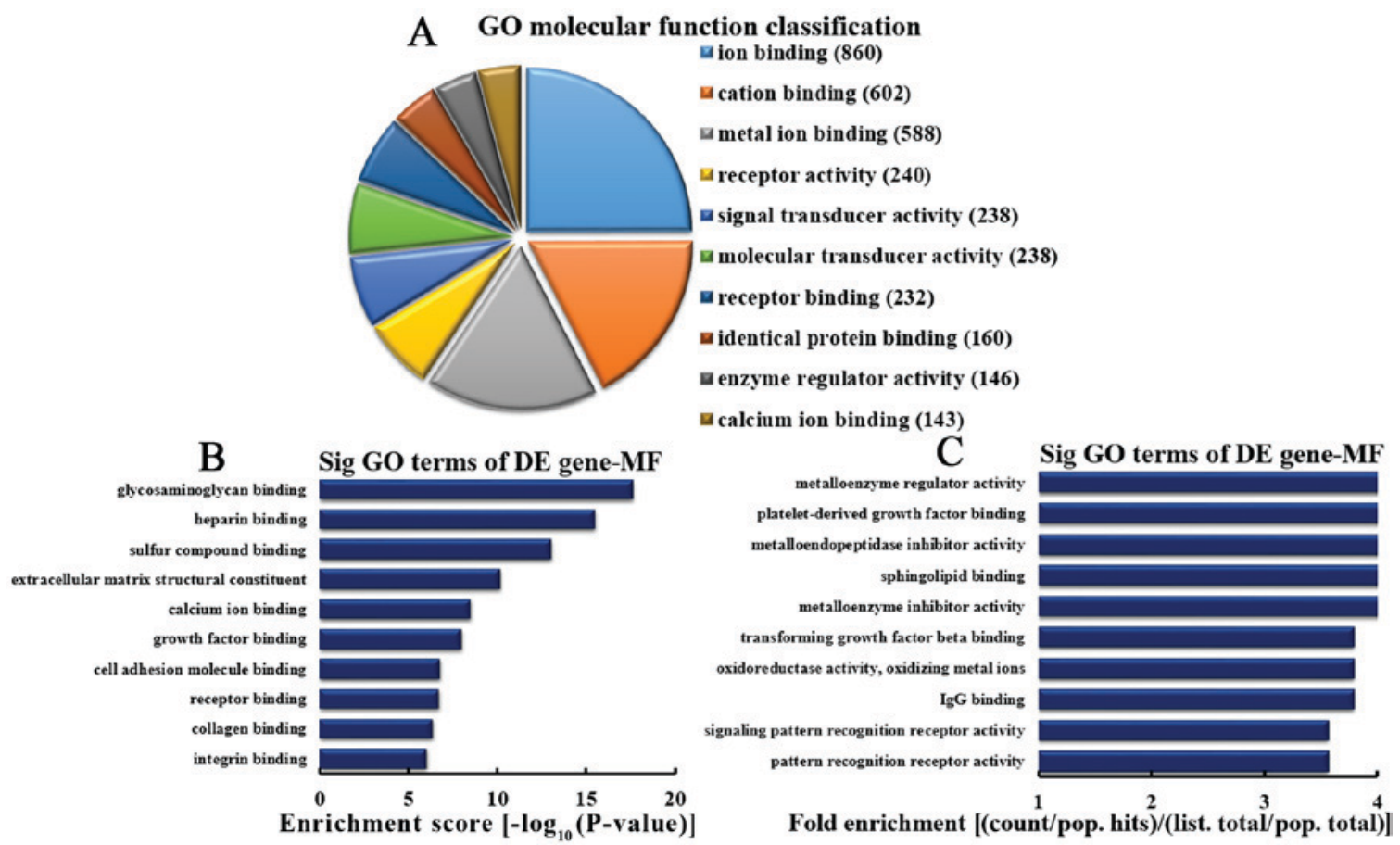

Figure 7. Analysis of downregulated mRNA involved in MFs. (A) MF classification based on the number of DE mRNA. (B) The most represented MFs based on the enrichment score. (C) The most represented MFs according to the fold enrichment. MF, molecular function; GO, gene ontology; DE, differentially expressed; Sig, significant.

included 2,138, 1,558, 1,026, 486, 482, 446, 425, 425, 408 and 389 mRNA involved in binding, protein binding, catalytic activity, small molecule binding, anion binding, hydrolase activity, nucleotide binding, nucleoside phosphate binding, carbohydrate derivative binding and transferase activity, respectively. Based on the enrichment score, the 10 most represented MFs were selected, as follows: Protein binding, catalytic activity, protein dimerization and heterodimerization activities, adenosine 5'-triphosphate and adenyl nucleotide binding, adenyl ribonucleotide binding, oxidoreductase activity acting on $\mathrm{CH}-\mathrm{OH}$ group of donors, oxidoreductase activity acting on $\mathrm{CH}-\mathrm{OH}$ group of donors or nicotinamide-adenine dinucleotide or nicotinamide-adenine dinucleotide phosphate acceptors, and mRNA with hydrolase activity (Fig. 6B). Based on the fold enrichment, the 10 most represented MFs included microtubule plus-end binding, acylglycerol O-acyltransferase activity, histone kinase activity, folic acid binding, WW domain binding, protein kinase B binding, racemase and epimerase activity, vitamin transporter activity, hydrolase activity on carbon-nitrogen (but not peptide) bonds in linear amidines, mitogen-activated protein kinase kinase binding and tau-protein kinase activity (Fig. 6C).

Analysis of downregulated mRNA implicated in MFs. Based on the number of downregulated mRNA associated with MFs, the 10 most represented MFs were as follows: $860,602,588,240,238,238,232,146,160$ and 143 mRNA involved in ion binding, cation binding, metal ion binding, receptor activity, signal transduction, molecular transduction, receptor binding, identical protein binding, enzyme regulatory activity and calcium ion binding, respectively (Fig. 7A). Based on enrichment scores, the 10 most represented MF were selected (Fig. 7B). These MFs included mRNA involved in the binding of glycosaminoglycan, heparin, sulfur binding, calcium ion, growth factor, cell adhesion molecules, receptor, collagen, integrin and extracellular matrix structural constituents. Based on fold enrichment analysis, the 10 most enriched MFs included the following: Metalloenzyme regulation, platelet-derived growth factor (PDGF) binding, metalloendopeptidase inhibition, sphingolipid binding, metalloenzyme inhibition, transforming growth factor $\beta$ binding, oxidoreductase activity, oxidizing metal ions, immunoglobulin $\mathrm{G}$ binding, signaling pattern recognition receptor and pattern recognition receptor activities (Fig. 7C).

Analysis of upregulated mRNA associated with CCs. According to the number of upregulated mRNA associated with CC, the 10 most enriched CCs were classified (Fig. 8A). A total of 2,211, 2,194, 2,129, 1,991, 1,901, 1,742, 1,728, 1,319, 1,255 and 1,217 mRNA were involved in intracellular, intracellular parts, organelles, membrane-bounded organelles, intracellular organelles, intracellular membrane-bounded organelles, cytoplasm, cytoplasmic parts, organelle parts and intracellular organelle parts, respectively. According to the enrichment score, the 10 most represented CCs were selected, as follows: Protein-DNA complexes, chromosomes, chromosomal parts, membrane-bounded organelles, extracellular organelles, extracellular membrane-bounded organelles, extracellular vesicular exosomes, DNA packaging complexes, organelle and extracellular region parts (Fig. 8B). Based on the fold enrichment, the 10 most represented CCs were selected: MCM complex, nuclear membrane parts, condensed nuclear chromosomes, centromeric regions, integral components of inner nuclear membranes, intrinsic components of inner nuclear membranes, outer kinetochores of condensed chromosomes, chromocenters, $\beta$-catenin 


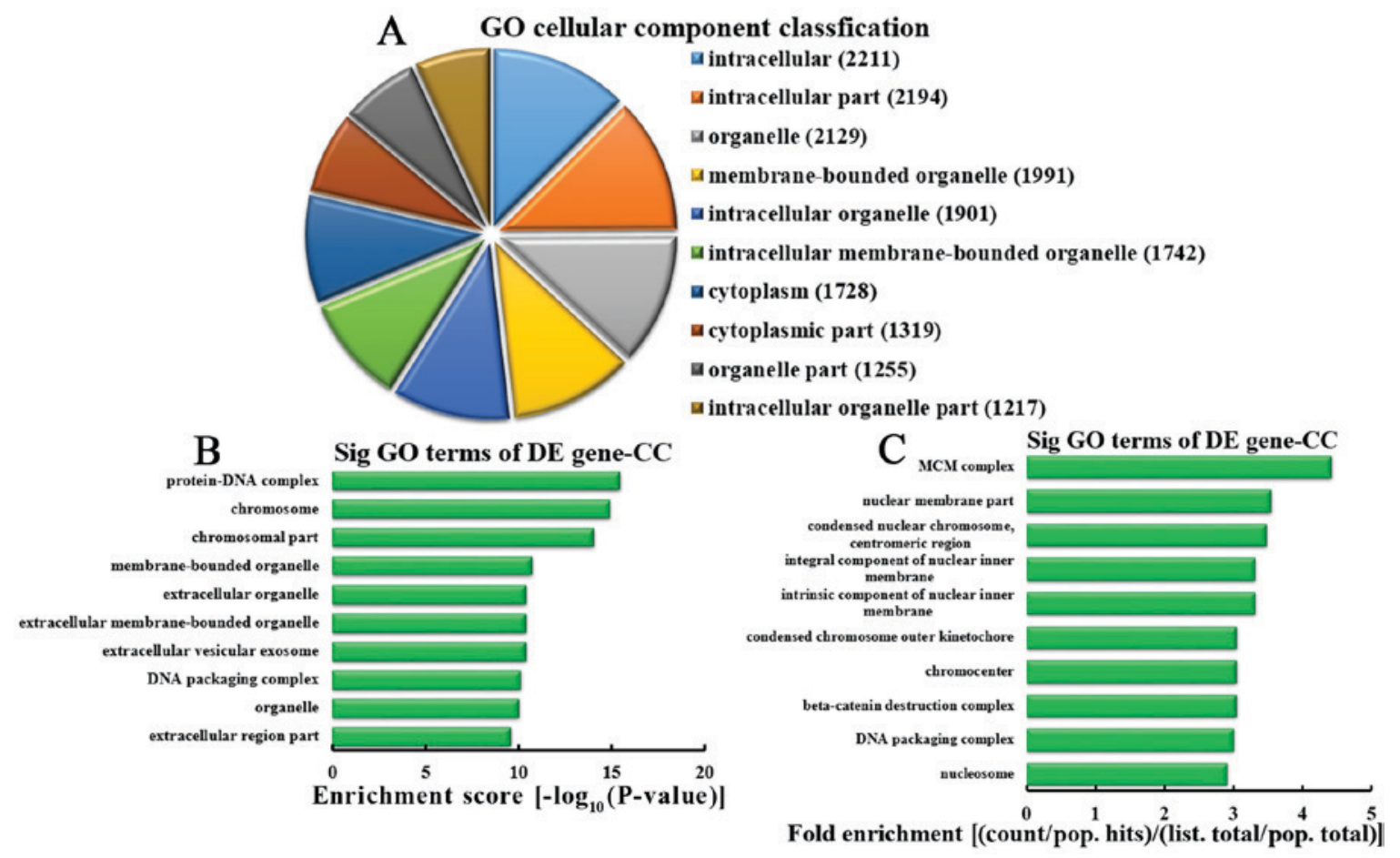

Figure 8. Analysis of upregulated mRNA involved in CCs. (A) CC classification based on the number of DE mRNA. (B) The most represented CCs based on the enrichment score. (C) The most represented CCs according to the fold enrichment. CC, cellular component; GO, gene ontology; DE, differentially expressed; Sig, significant.

destruction complexes, DNA packaging complexes and nucleosomes (Fig. 8C).

Analysis of downregulated mRNA associated with CCs. Based on the number of downregulated mRNA associated with CCs, the 10 most represented CCs were classified, as follows: 1,209 , 947, 820, 792, 763, 746, 726, 595, 514 and 498 mRNA involved in membranes, membrane parts, intrinsic components of membrane, integral components of membrane, cell periphery, plasma membranes, extracellular regions, parts of extracellular regions, mRNA involved in vesicles and mRNA involved in membrane-bound vesicles, respectively (Fig. 9A). According to enrichment score, the 10 most represented CCs were selected, including: Extracellular matrices, proteinaceous extracellular matrices, extracellular space, extracellular matrix parts, collagen trimers, plasma membrane parts, extracellular regions, cell periphery, cell surface and plasma membranes (Fig. 9B). Based on the fold enrichment, the 10 most represented CCs were selected, including fibrillar collagen trimers, interstitial matrices, collagen trimers, complexes of collagen trimers, parts of extracellular matrices, $\mathrm{M}$ bands, laminin complexes, basement membranes, fibrils and gap junctions (Fig. 9C).

Analysis of differentially expressed mRNA involved in signaling pathways. To further investigate the function of differentially expressed mRNA in CRC tissues between the $\mathrm{CRC}+\mathrm{m}$ group and $\mathrm{CRC}-\mathrm{m}$ group, the KEGG database was used for pathway analysis. The results demonstrated that the upregulated mRNA in the CRC $+\mathrm{m}$ group compared with the CRC $-m$ group were primarily associated with 58 signaling pathways. Downregulated mRNA in the $\mathrm{CRC}+\mathrm{m}$ group compared with the CRC - $\mathrm{m}$ group were primarily associated with 32 signaling pathways. Based on the enrichment score, the 10 most represented pathways among the upregulated mRNA were as follows: Alcoholism, DNA replication, cell cycle, systemic lupus erythematosus, propanoate and butanoate metabolism, bile secretion, mismatch repair, peroxisome proliferator-activated receptor signaling pathway and oocyte meiosis (Fig. 10A). Based on the enrichment score, the 10 most represented pathways among downregulated mRNA included complement and coagulation cascades, malaria, extracellular matrix-receptor interaction, cell adhesion molecules, glycosaminoglycan biosynthesis, staphylococcus aureus infection, phosphoinositide 3-kinase (PI3K)-Akt signaling pathway, focal adhesion, protein digestion and absorption, and dilated cardiomyopathy (Fig. 10B).

\section{Discussion}

CRC is major public health problem globally (18). Metastatic CRC develops on average within 3 years in $~ 50 \%$ of cases and lungs are a site of distant relapse $(19,20)$. Patients affected by metastatic CRC experience untimely diagnosis and poor prognosis. Therefore, it is necessary to determine the molecular mechanism underlying CRC with lung metastasis. In the present study, lncRNA and mRNA were differentially expressed in CRC tissues from patients with CRC + m compared with patients with CRC - m. GO and KEGG pathway analyses were conducted to predict the potential function and associated regulatory mechanism of mRNA in CRC tissues from patients with pulmonary metastasis.

A total of 7,632 lncRNA (3,574 upregulated and 4,058 downregulated) and 6,185 mRNA (3,394 upregulated and 2,791 downregulated) were differentially expressed in 


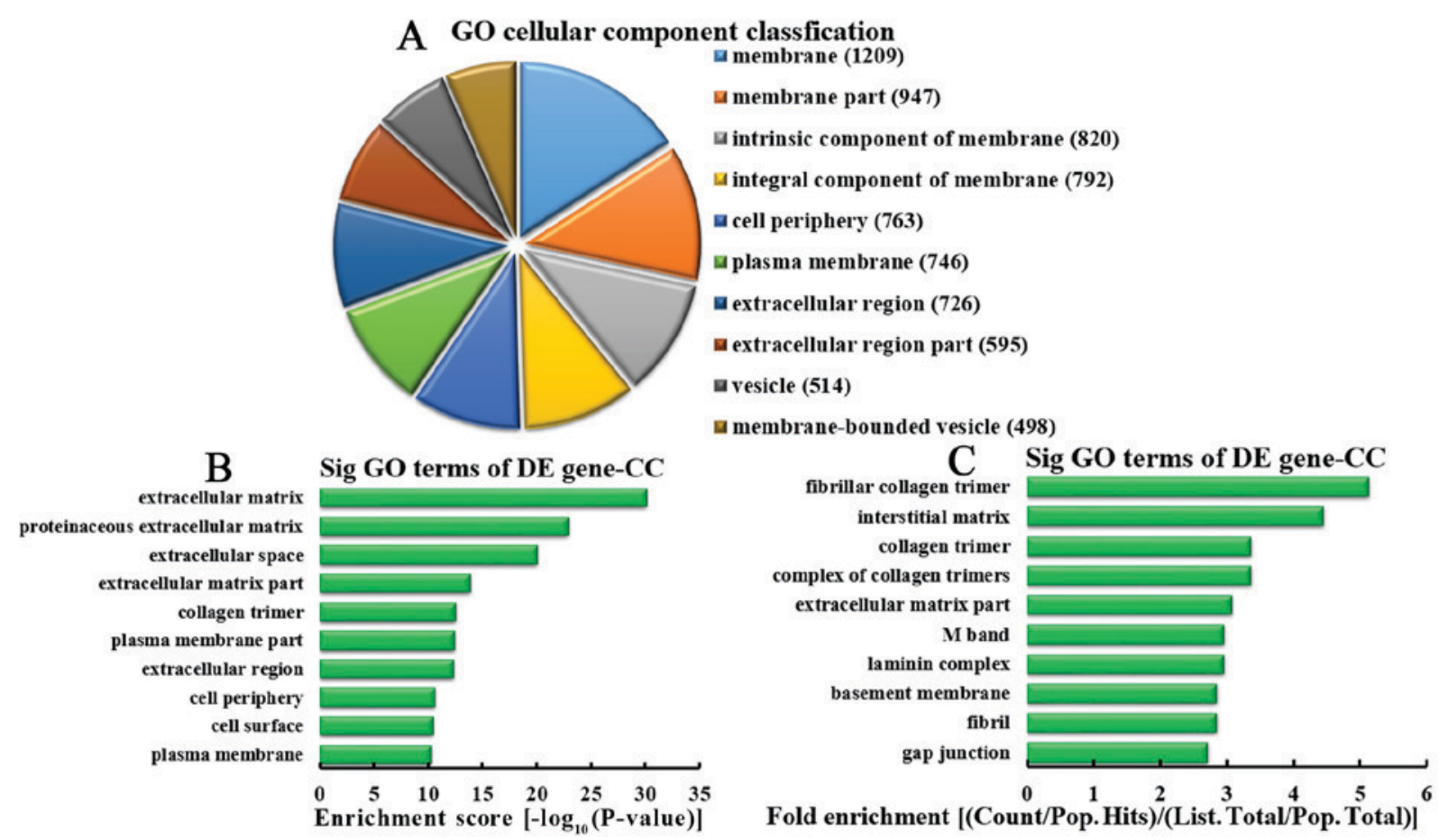

Figure 9. Analysis of downregulated mRNA involved in CCs. (A) CC classification based on the number of differentially expressed mRNA. (B) The most represented CCs based on the enrichment score. (C) The most represented CCs according to the fold enrichment. CC, cellular component; GO, gene ontology; DE, differentially expressed; Sig, significant.

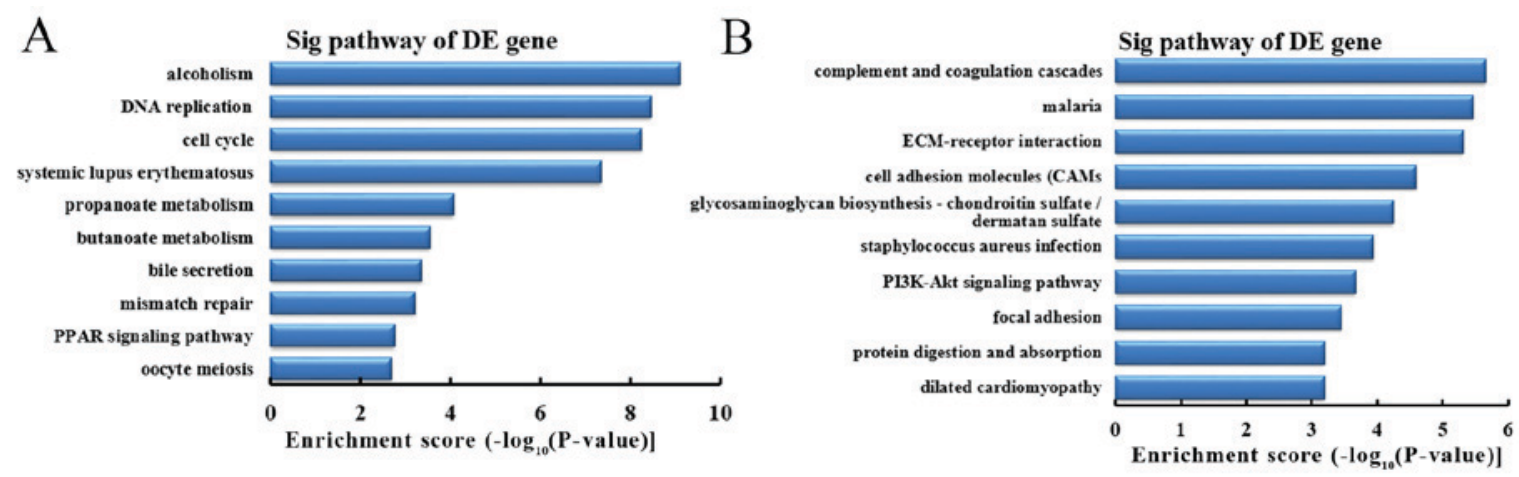

Figure 10. Kyoto Encyclopedia of Genes and Genomes pathway analysis of DE mRNA. The top 10 pathways enriched by (A) upregulated and (B) downregulated mRNA. DE, differentially expressed; Sig, significant; PPAR, peroxisome proliferator-activated receptor; ECM, extracellular matrix; PI3K, phosphoinositide 3-kinase.

CRC tissues from patients with CRC + m compared with samples from patient with $\mathrm{CRC}-\mathrm{m}$. A previous study indicated that 2,636 lncRNA were differentially expressed in the CRC tissues from patients with liver metastasis, including 1,600 upregulated and 1,036 downregulated, >2-fold compared with the CRC tissues without metastasis, and 1,584 mRNA (548 upregulated and 1,036 downregulated) were differentially expressed (8). It has also been reported that, in tumor and metastatic lymph node tissues, 53 lncRNA exhibited upregulated transcription levels and 337 lncRNA exhibited downregulated transcription, whereas 102 and 406 mRNA exhibited up- and downregulated expression levels, respectively (21). A total of 762 lncRNA (390 up- and 372 downregulated) were differentially expressed between CRC tissues and healthy tissues (22). To the best of our knowledge, the present study is the first to report differentially expressed lncRNA and mRNA in CRC tissues of patients with lung metastasis.

Using GO analysis in the present study, it was determined that the upregulated mRNA were associated with cell division (BPs), protein kinase B binding (MFs) and intracellular parts (CCs), while the downregulated mRNA were associated with cell adhesion, PDGF binding and membrane parts. It has been previously reported that upregulated differentially expressed genes were significantly enriched in cell division-associated processes in CRC tissues (23). SH2-domain-containing 5 inositol phosphatase has been hypothesized to serve a tumor initiating role by enhancing cell migration and invasion through protein kinase B activation in colonic epithelial cells (24). In silico analyses of deregulated proteins in the secretome of metastatic CRC cells demonstrated an increased abundance of 
proteins involved in cell adhesion, and host-related carinoembryonic antigen cell adhesion molecule 1 was hypothesized to promote metastasis of CRC (25). PDGF was highly expressed in early and late stages of primary CRCs and was elevated in platelets of patients with CRC $(26,27)$. Inhibition of mechanistic target of rapamycin kinase and PDGF prevented liver metastasis of CRC by regulating the tumor microenvironment (28).

In the present study, KEGG pathway analysis demonstrated that the Wnt signaling pathway was upregulated while the PI3K-Akt signaling pathway was downregulated in CRC $+\mathrm{m}$ tissues compared with the CRC - $m$ group. A previous study demonstrated that $90 \%$ of CRC cases occur due to the activation of the Wnt signaling pathway (29). The Wnt signaling pathway serves a role in regulating embryonic development, including body axis patterning, cell fate specification and cell migration (30). For example, fatty acid synthase knockdown attenuated the Wnt signaling pathway by downregulating distinctive genes, including Wnt5a, Wnt5b and Frizzled-2, which at least partly contributed to the decrease in metastasis of CRC (31). Hcrcn81 has been demonstrated to induce initiation and progression of carcinogenesis through regulation of the Wnt signaling pathway and serve a role in the carcinogenesis of CRC (32). It has been reported that Ras-related protein Rab-11A-family interacting proteins promoted migration and invasion of CRC cells through the upregulation of expression of matrix metallopeptidase 7 by activation of the PI3K/Akt signaling pathway (33). Ribonuclease inhibitor had been demonstrated to suppress proliferation and metastasis in CRC cells through inhibition of the PI3K/Akt pathway (34). Odontogenic ameloblast-associated protein suppresses human CRC by inactivating PI3K/Akt signaling (35). MicroRNA (miR)-92a is involved in lymph node metastasis in patients with CRC through the phosphatase and tensin homolog-regulated PI3K/Akt signaling pathway (36). miR-302a overexpression has been demonstrated to suppress proliferation and invasion of CRC cells by reducing the expression of associated proteins through the inhibition of the mitogen-activated protein kinase and PI3K/Akt signaling pathways (37). Therefore, the above studies combined with the results of the present study suggest that metastatic CRC is due to the interactive effects of multiple lncRNA engaged in modulation of a multi-gene system.

In conclusion, the present study identified a series of differentially expressed lncRNA and mRNA in patients with CRC with or without lung metastasis. The potential roles of IncRNA and mRNAs were predicted by bioinformatics analyses (38). The differentially expressed lncRNA identified in the present study may provide novel targets for elucidation of the molecular mechanisms underlying the development of metastatic $\mathrm{CRC}$ and for the diagnosis and prognosis of metastatic CRC.

\section{Acknowledgements}

The present study was supported by the fund of Yunling Scholar, the joint funds of Yannan Provincial Science and Technology Department and Kunming Medical University (grant nos. 2017FE467-038 and -130), Project of the Department of Health in Yunnan Province (grant nos. 2016NS002 and 2016NS003), and The General Joint Project of Yunnan Provincial Science and Technology Department and Kunming Medical University (grant no. 2015FB024).

\section{References}

1. Jemal A, Bray F, Center MM, Ferlay J, Ward E and Forman D: Global cancer statistics. CA Cancer J Clin 61: 69-90, 2011.

2. Shah SA, Haddad R, Al-Sukhni W, Kim RD, Greig PD, Grant DR, Taylor BR, Langer B, Gallinger S and Wei AC: Surgical resection of hepatic and pulmonary metastases from colorectal carcinoma. J Am Coll Surg 202: 468-475, 2006.

3. Lin PC, Lin JK, Lin CC, Wang HS, Yang SH, Jiang JK, Lan YT, Lin TC, Li AF, Chen WS and Chang SC: Carbohydrate antigen 19-9 is a valuable prognostic factor in colorectal cancer patients with normal levels of carcinoembryonic antigen and may help predict lung metastasis. Int J Colorectal Dis 27: 1333-1338, 2012.

4. Cejas P, López-Gómez M, Aguayo C, Madero R, de Castro Carpeño J, Belda-Iniesta C, Barriuso J, Moreno Garcia V, Larrauri J, López R, et al: KRAS mutations in primary colorectal cancer tumors and related metastases: A potential role in prediction of lung metastasis. PLoS One 4: e8199, 2009.

5. Mitry E, Guiu B, Cosconea S, Jooste V, Faivre J and Bouvier AM: Epidemiology, management and prognosis of colorectal cancer with lung metastases: A 30-year population-based study. Gut 59: 1383-1388, 2010.

6. Warwick R and Page R: Resection of pulmonary metastases from colorectal carcinoma. Eur J Surg Oncol 33 (Suppl 2): S59-S63, 2007.

7. Lee WS, Yun SH, Chun HK, Lee WY and Yun H: Clinical usefulness of chest radiography in detection of pulmonary metastases after curative resection for colorectal cancer. World J Surg 31: 1502-1506, 2007.

8. Chen D, Sun Q, Cheng X, Zhang L, Song W, Zhou D, Lin J and Wang W: Genome-wide analysis of long noncoding RNA (lncRNA) expression in colorectal cancer tissues from patients with liver metastasis. Cancer Med 5: 1629-1639, 2016.

9. ENCODE Project Consortium: An integrated encyclopedia of DNA elements in the human genome. Nature 489: 57-74, 2012.

10. Spizzo R, Almeida MI, Colombatti A and Calin GA: Long non-coding RNAs and cancer: A new frontier of translational research? Oncogene 31: 4577-4587, 2012.

11. Guttman M and Rinn JL: Modular regulatory principles of large non-coding RNAs. Nature 482: 339-346, 2012.

12. Cheetham SW, Gruhl F, Mattick JS and Dinger ME: Long noncoding RNAs and the genetics of cancer. Br J Cancer 108: 2419-2425, 2013.

13. Arase M, Horiguchi K, Ehata S, Morikawa M, Tsutsumi S, Aburatani H, Miyazono K and Koinuma D: Transforming growth factor- $\beta$-induced lncRNA-Smad7 inhibits apoptosis of mouse breast cancer JygMC(A) cells. Cancer Sci 105: 974-982, 2014.

14. Wang K, Long B, Zhou LY, Liu F, Zhou QY, Liu CY, Fan YY and Li PF: CARL lncRNA inhibits anoxia-induced mitochondrial fission and apoptosis in cardiomyocytes by impairing miR-539-dependent PHB2 downregulation. Nat Commun 5: 3596, 2014

15. Yang Y, Li H, Hou S, Hu B, Liu J and Wang J: The noncoding RNA expression profile and the effect of lncRNA AK126698 on cisplatin resistance in non-small-cell lung cancer cell. PLoS One 8: e65309, 2013.

16. Ye LC, Zhu X, Qiu JJ, Xu J and Wei Y: Involvement of long non-coding RNA in colorectal cancer: From benchtop to bedside (Review). Oncol Lett 9: 1039-1045, 2015.

17. Livak KJ and Schmittgen TD: Analysis of relative gene expression data using real-time quantitative PCR and the 2(-Delta Delta C(T)) method. Methods 25: 402-408, 2001.

18. Jemal A, Siegel R, Ward E, Hao Y, Xu J and Thun MJ: Cancer statistics, 2009. CA Cancer J Clin 59: 225-249, 2009.

19. Sargent DJ, Patiyil S, Yothers G, Haller DG, Gray R, Benedetti J, Buyse M, Labianca R, Seitz JF, O'Callaghan CJ, et al: End points for colon cancer adjuvant trials: Observations and recommendations based on individual patient data from 20,898 patients enrolled onto 18 randomized trials from the ACCENT group. J Clin Oncol 25: 4569-4574, 2007.

20. Guerrera F, Mossetti C, Ceccarelli M, Bruna MC, Bora G, Olivetti S, Lausi PO, Solidoro P, Ciccone G, Ruffini E, et al: Surgery of colorectal cancer lung metastases: Analysis of survival, recurrence and re-surgery. J Thorac Dis 8: 1764-1771, 2016.

21. Yang $\mathrm{P}, \mathrm{Xu} \mathrm{ZP}$, Chen $\mathrm{T}$ and $\mathrm{He} \mathrm{ZY}$ : Long noncoding RNA expression profile analysis of colorectal cancer and metastatic lymph node based on microarray data. Onco Targets Ther 9: 2465-2478, 2016. 
22. Xue Y, Ma G, Gu D, Zhu L, Hua Q, Du M, Chu H, Tong N, Chen J, Zhang $\mathrm{Z}$ and Wang $\mathrm{M}$ : Genome-wide analysis of long noncoding RNA signature in human colorectal cancer. Gene 556: 227-234, 2015.

23. Liang $\mathrm{B}, \mathrm{Li} \mathrm{C}$ and Zhao J: Identification of key pathways and genes in colorectal cancer using bioinformatics analysis. Med Oncol 33: 111, 2016

24. Hoekstra E, Das AM, Willemsen M, Swets M, Kuppen PJ, van der Woude CJ, Bruno MJ, Shah JP, Ten Hagen TL, Chisholm JD, et al Lipid phosphatase SHIP2 functions as oncogene in colorectal cancer by regulating PKB activation. Oncotarget 7: 73525-73540, 2016.

25. Arabzadeh A, Chan C, Nouvion AL, Breton V, Benlolo S, DeMarte L, Turbide C, Brodt P, Ferri L and Beauchemin N Host-related carcinoembryonic antigen cell adhesion molecule 1 promotes metastasis of colorectal cancer. Oncogene 32: 849-860, 2013.

26. Moench R, Grimmig T, Kannen V, Tripathi S, Faber M, Moll EM, Chandraker A, Lissner R, Germer CT, Waaga-Gasser AM and Gasser M: Exclusive inhibition of PI3K/Akt/mTOR signaling is not sufficient to prevent PDGF-mediated effects on glycolysis and proliferation in colorectal cancer. Oncotarget 7: 68749-68767, 2016.

27. Peterson JE, Zurakowski D, Italiano JE Jr, Michel LV, Connors S, Oenick M, D'Amato RJ, Klement GL and Folkman J: VEGF, PF4 and PDGF are elevated in platelets of colorectal cancer patients. Angiogenesis 15: 265-273, 2012

28. Yuge $\mathrm{R}$, Kitadai $\mathrm{Y}$, Shinagawa $\mathrm{K}$, Onoyama $\mathrm{M}$ Tanaka S, Yasui W and Chayama K: mTOR and PDGF pathway blockade inhibits liver metastasis of colorectal cancer by modulating the tumor microenvironment. Am J Pathol 185: $399-408,2015$

29. Subramaniyan B, Jagadeesan K, Ramakrishnan S and Mathan G: Targeting the interaction of Aurora kinases and SIRT1 mediated by Wnt signaling pathway in colorectal cancer: A critical review. Biomed Pharmacother 82: 413-424, 2016.

30. Liu Y, Huang D, Wang Z, Wu C, Zhang Z, Wang D, Li Z, Zhu T, Yang $S$ and Sun W: LMO2 attenuates tumor growth by targeting the Wnt signaling pathway in breast and colorectal cancer. Sci Rep 6: 36050, 2016.
31. Wang H, Xi Q and Wu G: Fatty acid synthase regulates invasion and metastasis of colorectal cancer via Wnt signaling pathway. Cancer Med 5: 1599-1606, 2016.

32. Chen Y, Jiang T, Shi L and He K: hcren81 promotes cell proliferation through Wnt signaling pathway in colorectal cancer. Med Oncol 33: 3, 2016

33. Xu CL, Wang JZ, Xia XP, Pan CW, Shao XX, Xia SL, Yang SX and Zheng B: Rab11-FIP2 promotes colorectal cancer migration and invasion by regulating PI3K/AKT/MMP7 signaling pathway. Biochem Biophys Res Commun 470: 397-404, 2016.

34. Tang Y,Liu P,Tian Y,Xu Y,RenF, Cui X andFan J: Overexpression of ribonuclease inhibitor defines good prognosis and suppresses proliferation and metastasis in human colorectal cancer cells via PI3K/AKT pathway. Clin Transl Oncol 17: 306-313, 2015.

35. Yu M, Mu Y, Qi Y, Qin S, Qiu Y, Cui R and Zhong M: Odontogenic ameloblast-associated protein (ODAM) inhibits human colorectal cancer growth by promoting PTEN elevation and inactivating PI3K/AKT signaling. Biomed Pharmacother 84: 601-607, 2016.

36. Ke TW, Wei PL, Yeh KT, Chen WT and Cheng YW: MiR-92a promotes cell metastasis of colorectal cancer through PTEN-mediated PI3K/AKT pathway. Ann Surg Oncol 22: 2649-2655, 2015.

37. Wei ZJ, Tao ML, Zhang W, Han GD, Zhu ZC, Miao ZG, Li JY and Qiao ZB: Up-regulation of microRNA-302a inhibited the proliferation and invasion of colorectal cancer cells by regulation of the MAPK and PI3K/Akt signaling pathways. Int J Clin Exp Pathol 8: 4481-4491, 2015.

38. Huang M,Zhong Z,Lv M,Shu J,Tian Q and Chen J: Comprehensive analysis of differentially expressed profiles of lncRNAs and circRNAs with associated co-expression and ceRNA networks in bladder carcinoma. Oncotarget 7: 47186-47200, 2016.

This work is licensed under a Creative Commons Attribution-NonCommercial-NoDerivatives 4.0 International (CC BY-NC-ND 4.0) License. 